\title{
Bifurcations of Piecewise Smooth Vector Fields via Geometric Singular Perturbations
}

\author{
Durval J. Tonon \\ Instituto de Matemática e Estatística, UFG \\ 74001-970, Goiânia, GO \\ E-mail: djtonon@ufg.br \\ Tiago de Carvalho \\ Departamento de Matemática, Faculdade de Ciências, UNESP \\ 17033-360, Bauru, SP \\ E-mail: tcarvalho@fc.unesp.br
}

\begin{abstract}
We deal with piecewise smooth vector fields on the plane and prove that the analysis of their local behavior around certain typical singularities can be treated via singular perturbation theory. In fact, after a regularization of a such system and a blow-up we are able to bring out some results that bridge the space between piecewise smooth vector fields presenting typical singularities and singularly perturbed smooth systems.
\end{abstract}

Keyword: geometric singular perturbation, piecewise smooth vector fields, bifurcation.

\section{Introduction}

This work fits within the geometric study of singular perturbation problems expressed by vector fields on $\mathbb{R}^{2}$. We study the phase portraits of certain non-smooth (piecewise smooth) planar vector fields having a curve $\Sigma$ as the discontinuity set. We present some results in the framework developed by Sotomayor and Teixeira in [7] (and extended in [6]) and establish a bridge between those systems and the fundamental role played by the geometric singular perturbation theory. This transition was introduced in papers like [1] and [4], in dimensions 2 and 3 respectively. Results in this context can be found in [5]. We deal with non-smooth vector fields presenting structurally unstable configurations and we prove that these structurally unstable configurations are carried over the geometric singular perturbation problem associated.

\subsection{Setting the problem}

Let $\mathcal{U} \subseteq \mathbb{R}^{2}$ be an open set, $(0,0) \in \mathcal{U}$ and $\Sigma=\{(x, y) \in \mathcal{U} \mid x=0\}$. Clearly $\Sigma$ is the separating boundary of the regions $\Sigma_{+}=\{(x, y) \in \mathcal{U} \mid x \geq 0\}$ and $\Sigma_{-}=\{(x, y) \in \mathcal{U} \mid x \leq 0\}$. Denote by $\mathfrak{X}^{r}$ the space of $C^{r}$-vector fields on a compact set $K \subset \mathcal{U}$ endowed with the $C^{r}$-topology with $r \geq 1$. Consider $Z: K \backslash \Sigma \rightarrow \mathbb{R}^{2}$ such that

$$
Z(x, y)=\left\{\begin{array}{lll}
X(x, y), & \text { for } \quad(x, y) \in \Sigma_{+}, \\
Y(x, y), & \text { for } \quad(x, y) \in \Sigma_{-},
\end{array}\right.
$$

where $X=\left(h_{1}, g_{1}\right), Y=\left(h_{2}, g_{2}\right) \in \mathfrak{X}^{r}$. We denote $Z=(X, Y)$ and distinguish the following regions on $\Sigma$ :

- Sewing Region $\Sigma_{\mathbf{1}}$ : one smooth vector field $X$ (resp., $Y$ ) push orbits towards $\Sigma_{1} \subset \Sigma$ and the other smooth vector field $Y$ (resp., $X$ ) push the orbits away form $\Sigma_{1} \subset \Sigma$. 
- Escaping Region $\boldsymbol{\Sigma}_{\mathbf{2}}$ : the two smooth vector fields $X$ and $Y$ from both sides out of $\Sigma_{2} \subset \Sigma$ push orbits away from $\Sigma_{2} \subset \Sigma$.

- Sliding Region $\boldsymbol{\Sigma}_{3}$ : the two smooth vector fields $X$ and $Y$ from both sides out of $\Sigma_{3} \subset \Sigma$ push orbits towards $\Sigma_{3} \subset \Sigma$.

Definition 1. The sliding vector field associated to $Z=(X, Y)$ is the vector field $Z^{\text {s }}$ tangent to $\Sigma_{3}$ and defined at $q \in \Sigma_{3}$ by $Z^{s}(q)=m-q$ with $m$ being the point of the segment joining $q+X(q)$ and $q+Y(q)$ such that $m-q$ is tangent to $\Sigma_{3}$ (see Figure 1).

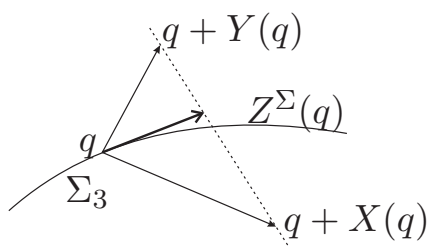

Figura 1: Filippov's convention.

Observe that if $q \in \Sigma_{2}$ for $Z=(X, Y)$ then $q \in \Sigma_{3}$ for $-Z=(-X,-Y)$. Therefore we can define the escaping vector field on $\Sigma_{2}$ associated to $Z$ by $Z^{e}=-(-Z)^{s}$. The sewing vector field associated to $Z$ is the vector field $Z^{w}$ defined in $q \in \Sigma_{1}$ as an arbitrary convex combination of $X(q)$ and $Y(q)$, i.e., $Z^{w}(q)=\lambda X(q)+(1-\lambda) Y(q)$ where $\lambda \in[0,1]$. In what follows we use the notation $Z^{\Sigma}$ for all these cases.

Let $\Omega^{r}$ be the space of vector fields $Z: K \rightarrow \mathbb{R}^{2}$ such that $Z(q)$ satisfies (1) when $q \in K \backslash \Sigma$ and $Z(q)=Z^{\Sigma}(q)$ when $q \in \Sigma$. The vector field $Z$ will be called a piecewise smooth vector field (PSVF for short). The basic results of differential equations, in this context, were stated by Filippov in [3].

The trajectories of $Z$ in some points of $K$ may present behaviors that need to be distinguished. These points will be called $\boldsymbol{\Sigma}$-singularities of $Z$ and are divided in two subsets: $\Sigma^{t}$ and $\Sigma^{p}$. Any $q \in \Sigma^{p}$ is called a pseudo equilibrium of $Z$ if $q$ is an equilibrium point of $Z^{\Sigma}$. Any $q \in \Sigma^{t}$ is called a tangential singularity if the trajectory of $X$ (resp., $Y$ ) by $q$ is tangent to $\Sigma$. A tangential singularity $q \in \Sigma^{t}$ is a $\Sigma$-fold point of $X$ (resp., $Y$ ) if the tangency is quadratic. Moreover, when the trajectory of $X$ (resp., $Y$ ) by the $\Sigma$-fold point belongs to $\Sigma_{+}$(resp., $\Sigma_{-}$) we call it a visible $\Sigma$-fold point. When the trajectory of $X$ (resp., $Y$ ) by the $\Sigma$-fold point belongs to $\Sigma_{-}$(resp., $\Sigma_{+}$) we call it an invisible $\Sigma$-fold point.

\section{Regularization}

In this section we present the concept of $\epsilon$-regularization of PSVFs. It was introduced by Sotomayor and Teixeira in [7]. The method consists in the analysis of the regularized vector field which is a smooth approximation of the original PSVF. Using this process we get a 1-parameter family of vector fields $Z_{\epsilon} \in \mathfrak{X}^{r}$ such that for each $\epsilon_{0}>0$ fixed $Z_{\epsilon_{0}}$ is equal to $X$ (resp., $Y$ ) in all points of $\Sigma_{+}$(resp., $\Sigma_{-}$) whose distance to $\Sigma$ is bigger than $\epsilon_{0}$.

Definition 2. $A C^{\infty}$-function $\varphi: \mathbb{R} \longrightarrow \mathbb{R}$ is a transition function if $\varphi(x)=-1$ for $x \leqslant-1$, $\varphi(x)=1$ for $x \geqslant 1$ and $\varphi^{\prime}(x)>0$ if $x \in(-1,1)$. The $\epsilon$-regularization of $Z=(X, Y)$ is the 1 -parameter family $Z_{\epsilon} \in \mathfrak{X}^{r}$ given by

$$
Z_{\epsilon}(q)=\left(\frac{1}{2}+\frac{\varphi_{\epsilon}(f(q))}{2}\right) X(q)+\left(\frac{1}{2}-\frac{\varphi_{\epsilon}(f(q))}{2}\right) Y(q) .
$$

with $\varphi_{\epsilon}(x)=\varphi(x / \epsilon)$, for $\epsilon>0$. 


\section{Singular Perturbations}

Definition 3. Let $U \subseteq \mathbb{R}^{2}$ be an open subset and take $\epsilon \geqslant 0$. A singular perturbation problem in $U$ (SP-Problem) is a differential system which can be written like

$$
x^{\prime}=d x / d \tau=l(x, y, \epsilon), \quad y^{\prime}=d y / d \tau=\epsilon m(x, y, \epsilon)
$$

or equivalently, after the time re-scaling $t=\epsilon \tau$

$$
\epsilon \dot{x}=\epsilon d x / d t=l(x, y, \epsilon), \quad \dot{y}=d y / d t=m(x, y, \epsilon),
$$

with $(x, y) \in U$ and $l, m$ smooth in all variables.

The understanding of the phase portrait of the vector field associated to a $\mathrm{SP}$-problem is the main goal of the geometric singular perturbation theory. System (3) is called the fast system, and (4) the slow system of the SP-problem. Observe that for $\epsilon>0$ the phase portraits of the fast and the slow systems coincide. For $\epsilon=0$, let $\mathcal{S}$ be the set $\mathcal{S}=\{(x, y): l(x, y, 0)=0\}$ of all singular points of (3). We call $\mathcal{S}$ the slow critical manifold of the SP-problem and it is important to notice that equation (4) defines a dynamical system, on $\mathcal{S}$, called the reduced problem:

$$
l(x, y, 0)=0, \quad \dot{y}=m(x, y, 0) .
$$

Combining results on the dynamics of these two limiting problems, with $\epsilon=0$, one obtains information on the dynamics of $Z_{\epsilon}$ for small values of $\epsilon$. We refer to [2] for an introduction to the general theory of singular perturbations.

\section{Statement of the main result}

The main result of this work is the following:

Theorem 1. Consider $Z(x, y)=Z_{\mu}(x, y)=\left(X_{\mu}(x, y), Y_{\mu}(x, y)\right) \in \Omega^{r}$ where either $\mu=\lambda \in \mathbb{R}$ or $\mu=(\lambda, \varepsilon) \in \mathbb{R}^{2}$. Consider that $q=\left(x_{q}, y_{q}\right) \in \Sigma$ is a $\Sigma$-fold point of both $X_{\mu}$ and $Y_{\mu}$ when $\mu=0$ or $\mu=(0,0)$. Then there exists a singular perturbation problem

$$
\theta^{\prime}=\alpha(r, \theta, y, \mu), y^{\prime}=r \beta(r, \theta, y, \mu),
$$

with $r \geq 0, \theta \in(\pi / 4,3 \pi / 4), y \in \Sigma, \alpha$ and $\beta$ of class $C^{r}$ such that the following statements holds:

(a) For all small neighborhood $U$ of $q$ in $\Sigma$ the region $\left(\Sigma_{2} \cup \Sigma_{3}\right) \cap\left(U-\left\{y_{q}\right\}\right)$ is homeomorphic to the slow critical manifold $\alpha(0, \theta, y, \mu)=0$ of (5) where $y \in\left(U-\left\{y_{q}\right\}\right)$.

(b) The vector field $Z^{\Sigma}$, on $\left(\Sigma_{2} \cup \Sigma_{3}\right) \cap\left(U-\left\{y_{q}\right\}\right)$, and the reduced problem of (5), with $y \in\left(U-\left\{y_{q}\right\}\right)$, are topologically equivalent.

(c) The slow critical manifold $\alpha(0, \theta, y, 0)=0$ of (5), where $y=y_{q}$, has just an horizontal component, i.e., $\alpha\left(0, \theta, y_{q}, 0\right)=0$ can be identified with $\left\{(\theta, y) \mid \theta \in(\pi / 4,3 \pi / 4), y=y_{q}\right\}$. Moreover, this configuration is structurally unstable.

The unfolding of (5) produces the same topological behaviors as the unfolding of the corresponding normal forms of $Z_{\lambda}$ presented in Table 1 and in Equation (7).

Observe that Theorem 1 generalizes Theorem 1.1 of [5], because here we allow that both $X$ and $Y$ can have a quadratic contact with $\Sigma$ at a point $q$. In fact, if at most one of the vector fields $X$ or $Y$ presents a quadratic contact with $\Sigma$ at $q$ then Theorem 1.1 of [5] says that there exists a singular perturbation problem such that the sliding region is homeomorphic to the slow critical manifold and the sliding vector field is topologically equivalent to the reduced problem. 


\section{Proof of the main result}

In this section we analyze the dynamics of a PSVF around a point $q$ which is a $\Sigma$-fold point of both $X$ and $Y$. We say that $q$ is a Fold-Fold singularity of $Z \in \Omega^{r}$.

The content of this section proves Theorem 1 .

We divide the fold-fold singularities in three types according with the contact between $X, Y$ and $\Sigma$ :

(a) Elliptic case: $q$ is an invisible $\Sigma$-fold point of both $X$ and $Y$. See Figure 2 (a).

(b) Hyperbolic case: $q$ is a visible $\Sigma$-fold point of both $X$ and $Y$. See Figure 2 (b).

(c) Parabolic case: $q$ is an invisible $\Sigma$-fold point of $X$ (resp., $Y$ ) and a visible $\Sigma$-fold point of both $Y$ (resp., $X$ ). See Figure 2 (c).

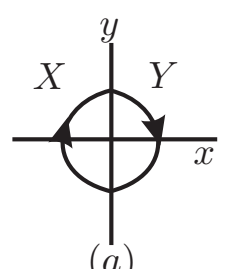

(a)

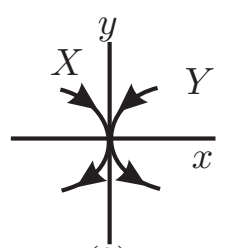

(b)

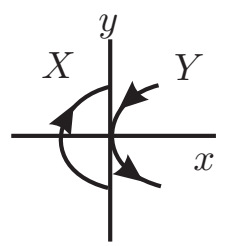

(c.1)

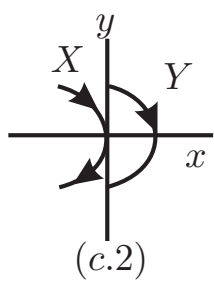

$(c .2)$

Figura 2: Fold-fold singularities.

Consider the parameter $\lambda \in(-1,1)$. Specific topological normal forms of the hyperbolic and parabolic fold-fold singularities are given in Table 1. A specific topological normal form of the elliptic fold-fold singularity is given in Subsection 5.3.

\begin{tabular}{|c|c|c|}
\hline Hyperbolic & Parabolic - Kind 1 & Parabolic - Kind 2 \\
\hline \hline$X_{\lambda}(x, y)=(y-\lambda,-1)$ & $X_{\lambda}(x, y)=(y-\lambda, 2)$ & $X_{\lambda}(x, y)=(y-\lambda,-2)$ \\
$Y(x, y)=(-y,-1)$ & $Y(x, y)=(-y,-1)$ & $Y(x, y)=(y,-1)$ \\
\hline
\end{tabular}

Tabela 1:

\subsection{Hyperbolic Case}

Consider the topological normal form of the hyperbolic fold-fold singularity given in Table 1. The regularized vector field is given by

$$
\dot{x}=-\frac{\lambda}{2}+\varphi\left(\frac{x}{\epsilon}\right)\left(\frac{-\lambda+2 y}{2}\right), \dot{y}=-1 .
$$

By the polar blow up we get

$$
r \dot{\theta}=\sin \theta\left(\frac{\lambda}{2}+\varphi(\cot \theta) \frac{\lambda-2 y}{2}\right), \dot{y}=-1 .
$$

Putting $r=0$ the fast dynamics is determined by the system

$$
\theta^{\prime}=\sin \theta\left(\frac{\lambda}{2}+\varphi(\cot \theta)\left(\frac{\lambda-2 y}{2}\right)\right), \quad y^{\prime}=0 ;
$$

and the slow dynamics on the slow critical manifold is determined by the reduced system 


$$
\frac{\lambda}{2}+\varphi(\cot \theta)\left(\frac{\lambda-2 y}{2}\right)=0, \quad \dot{y}=-1 .
$$

In this case we obtain the explicit expression for the slow manifold:

$$
y(\theta)=\frac{\lambda(1+\varphi(\cot \theta))}{2 \varphi(\cot \theta)} .
$$

Observe that, the slow critical manifold $y(\theta)$ is not defined for $\theta_{0}$ such that $\varphi\left(\cot \theta_{0}\right)=0$. So, for $\lambda \neq 0, y(\theta)$ has two branches and satisfies:

(a) $\lim _{\theta \longrightarrow \theta_{0}^{-}} y(\theta)=-\infty$ for $\lambda<0$ and $\lim _{\theta \longrightarrow \theta_{0}^{-}} y(\theta)=+\infty$ for $\lambda>0$;

(b) $\lim _{\theta \longrightarrow \theta_{0}^{+}} y(\theta)=+\infty$ for $\lambda<0$ and $\lim _{\theta \longrightarrow \theta_{0}^{+}} y(\theta)=-\infty$ for $\lambda>0$.

(c) For $\lambda=0$ the slow critical manifold is given implicitly by $y \varphi(\cot \theta)=0$, that is, $\{(\theta, y) \mid \theta=$ $\left.\theta_{0}\right\} \cup\{(\theta, y) \mid y=0\}$ is the slow manifold.
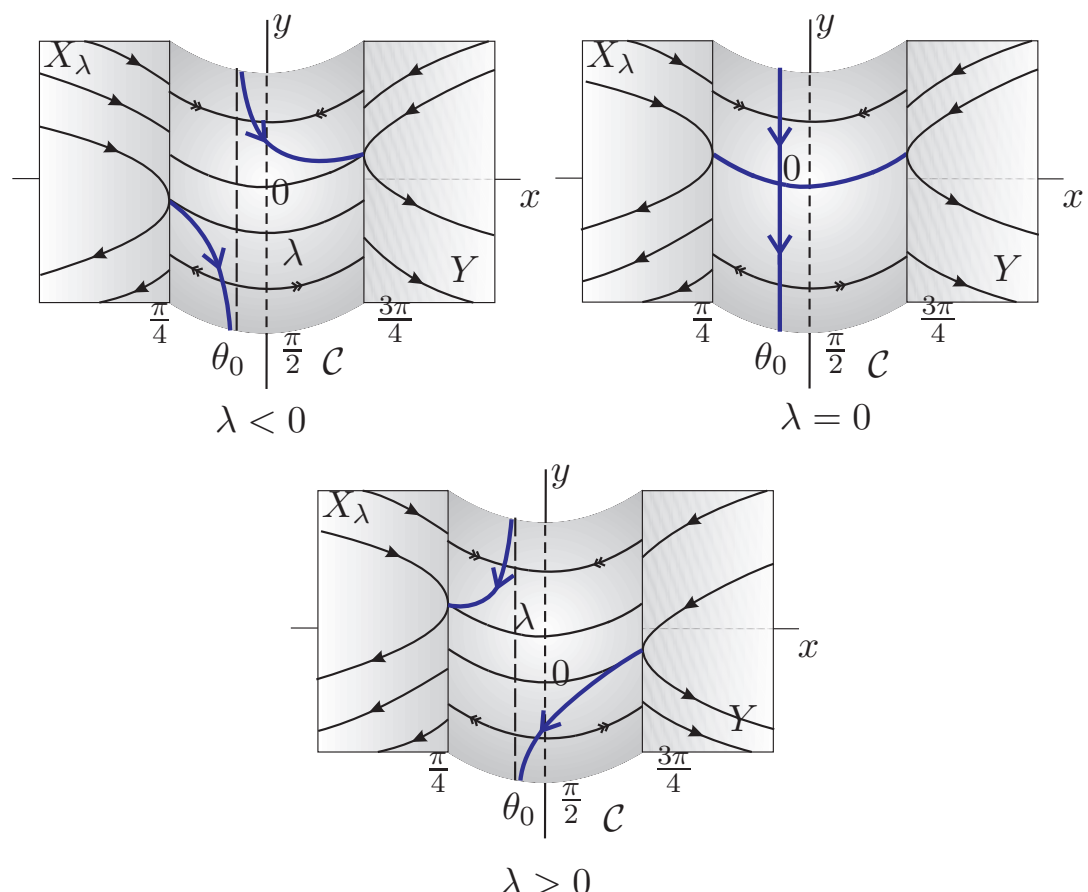

Figura 3: Slow manifold depending on the parameter $\lambda$.

The dynamics on the slow critical manifold is given by $\dot{y}=-1$. Therefore, there are not critical points. See Figure 3.

\subsection{Parabolic case}

Consider the topological normal form of the parabolic-Kind 1 (the analysis of the Kind 2 is analogous) fold-fold singularity given in Table 1.

Repeating the analysis of Section 5.1 we obtain that the fast dynamics is determined by the system

$$
\theta^{\prime}=\sin \theta\left(\frac{\lambda}{2}+\varphi(\cot \theta)\left(\frac{\lambda-2 y}{2}\right)\right), \quad y^{\prime}=0 ;
$$


and the slow dynamics on the slow critical manifold is determined by the reduced system

$$
\lambda+\varphi(\cot \theta)(\lambda-2 y)=0, \quad \dot{y}=\frac{1}{2}+\frac{3}{2} \varphi(\cot \theta) .
$$

The dynamics on the slow critical manifold is given by $\dot{y}=1 / 2+3 \varphi(\cot \theta) / 2$. For $\lambda<0$ (respectively $\lambda>0$ ) it presents a repeller critical point (respectively an attractor critical point). For $\lambda=0$ the slow critical manifold is composed by two branches. See Figure 4 .
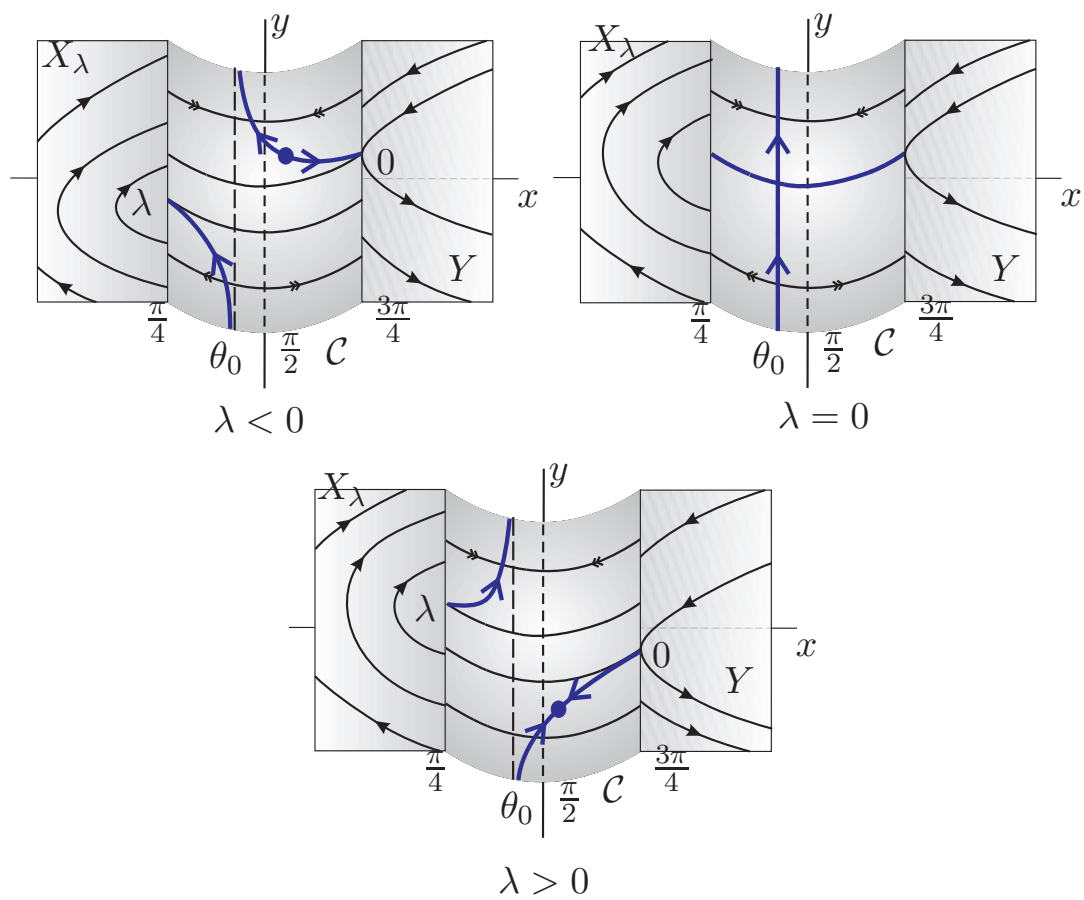

Figura 4: Bifurcation Diagram of the Parabolic-Kind 1 Fold-Fold Singularity.

\subsection{Elliptic case}

In this case, associated to the PSVF there exist a first return map $\psi_{Z}(p)$. A similar analysis to that one presented in Section 5.1 can be performed and we conclude that a normal form of the PSVF with this behavior is

$$
Z_{\lambda, \varepsilon}(x, y)= \begin{cases}X_{\lambda}(x, y)=(y-\lambda, 1), & \text { if }(x, y) \in \Sigma_{+} \\ Y_{\varepsilon}(x, y)=\left(h_{2}^{\varepsilon}(x, y), g_{2}^{\varepsilon}(x, y)\right), & \text { if }(x, y) \in \Sigma_{-}\end{cases}
$$

where the smooth function $h_{2}^{\varepsilon}$ and $g_{2}^{\varepsilon}$ satisfies the conditions $(a),(b)$ and $(c)$ below:

(a) $h_{2}^{\varepsilon}(0)=0$;

(b) $h_{2}^{\varepsilon}(0) \frac{d}{d x} h_{2}^{\varepsilon}(0)+g_{2}^{\varepsilon}(0) \frac{d}{d y} h_{2}^{\varepsilon}(0) \neq 0$;

(c) $\int_{0}^{t^{*}} g_{2}^{\varepsilon}(s) d s=(2+\varepsilon) y+O\left(y^{2}\right)$.

The hypotheses expressed in Item $(d)$ above give us sufficient conditions to get the unfolding of the first return map $\psi_{Z_{\lambda}}$. 


\subsection{Proof of Theorem 1}

Consider a PSVF $Z_{\lambda}=\left(X_{\lambda}, Y\right)$ where $\lambda \in \mathbb{R}$ is a parameter. If with the variation of $\lambda \in(-\varepsilon, \varepsilon)$, the following behaviors are observable then we consider that $Z_{\lambda}$ presents a bifurcation, where $\varepsilon>0$ and small. Consider $\lambda^{+} \in(0, \varepsilon)$ and $\lambda^{-} \in(-\varepsilon, 0)$. The behaviors are:

(i) A change of stability on $\Sigma$, i.e., where $Z_{\lambda^{+}}$has a sliding region $\Sigma_{3}$ the PSVF $Z_{\lambda^{-}}$has an escaping region $\Sigma_{2}$.

(ii) A change of stability on $\dot{y}_{\lambda}$, i.e., there are components of $\Sigma$ such that the induced flow on the slow manifold is such that $\dot{y}_{\lambda^{+}}>0$ and $\dot{y}_{\lambda^{-}}<0$.

(iii) A change of stability of the $\Sigma$-singularity, i.e., $Z_{\lambda^{+}}$presents an attractor equilibrium and $Z_{\lambda^{-}}$presents a repeller requilibrium.

(iv) A change of orientation on $\Sigma_{1}$ (the sewer region), i.e., $Z_{\lambda^{+}}$and $Z_{\lambda^{-}}$presents distinct orientations on $\Sigma_{1}$.

In face of these previous observations, Theorem 1 follows straightforward from Section 5.

Note that, as we give the topological behavior of the cases $\lambda<0, \lambda=0$ and $\lambda>0$ it is easy to construct the bifurcation diagram of (5) when $\lambda \in \mathbb{R}$.

The case $\mu=(\lambda, \varepsilon) \in \mathbb{R}^{2}$ is used in Subsection 5.3 with analogous results.

Acknowledgments. The first author is partially supported by FAPESP-BRAZIL, grant 2012/00481-6. This work is partially realized at UFG/Brazil as part of projects number 35796 and 35797.

\section{Referências}

[1] C.A. Buzzi, P.R. DA Silva And M. A. Teixeira, A singular approach to discontinuous vector fields on the plane, Journal of Differential Equations, 231 (2006), 633-655.

[2] N. Fenichel, Geometric singular perturbation theory for ordinary differential equations, Journal of Differential Equations 31 (1979), 53-98.

[3] A.F. Filippov, Differential equations with discontinuous righthand sides, Mathematics and its Applications (Soviet Series), Kluwer Academic Publishers-Dordrecht, 1988.

[4] J. Llibre, P.R. Silva And M.A. Teixeira, Regularization of discontinuous vector fields via singular perturbation, J. Dynam. Differential Equation 19 (2006), 309-331.

[5] J. Llibre, P.R. Silva And M.A. Teixeira, Sliding vector fields via slow-fast systems, Bulletin of the Belgian Mathematical Society Simon Stevin 15-5 (2008), 851-869.

[6] J. Llibre and M.A. Teixeira, Regularization of discontinuous vector fields in dimension three, Discrete Cont. Dynam. Systems 3 (1997), 235-241.

[7] J. Sotomayor and M.A. TeixeIra, Regularization of discontinuous vector fields, International Conference on Differential Equations, Lisboa (1996), 207-223. 